\title{
Algoritmo de tratamiento en las secuelas cicatrizales posquemadura en codo
}

\author{
Treatment algorithm in post-elbow burn scar sequelae
}

Esteban Elena Scarafoni', Héctor Martínez' ${ }^{1}$, Christian Vidaurre' ${ }^{1}$, Carlos E. Sereday²

\section{RESUMEN}

Introducción. Las retracciones cicatrizales secundarias a quemaduras representan un desafío para el cirujano plástico debido a la variabilidad en su complejidad y presentación. La pérdida de movimiento del codo conlleva una limitación funcional con impacto directo en la calidad de vida, por lo que la indicación quirúrgica se hace necesaria. La gran variedad de tácticas quirúrgicas plantean la necesidad de sistematizar el tratamiento de las retracciones cicatrizales.

Objetivo. El objetivo de esta presentación es la aplicación de la sistemática en el tratamiento de la secuela posquemadura de codo en el Servicio de Cirugía Plástica del Hospital de Quemados del Gobierno de la Ciudad de Buenos Aires durante los últimos 10 años.

Material y métodos. Mediante un diseño observacional, descriptivo y retrospectivo, se realizó el presente trabajo sobre pacientes con diagnóstico de retracción cicatrizal posuemadura en codo de agosto de 2008 a agosto de 2018. El criterio de inclusión fue pacientes entre 3 y 65 años sometidos a cirugías reconstructivas para la liberación de contracturas del codo posteriores a una quemadura durante el período mencionado. Se realizó una revisión de las historias clínicas y se evaluaron los casos a través del examen físico y de documentación fotográfica, y se analizaron diferentes variables: sexo, edad, comorbilidades, clasificación de gravedad, grado de retracción pre- y posoperatoria, tipo de reconstrucción empleada y complicaciones. Las variables mencionadas se registraron en tablas.

Resultados. Treinta y cuatro pacientes operados, 20 mujeres y 14 hombres. El rango de edad fue de 3 hasta los 50 años, con una media de 21,2 años. La causa más frecuente de quemadura fue el agua caliente. El grado de retracción fue leve en $41,17 \%$ ( 14 casos), moderada en $47,05 \%$ y severa en $11,7 \%$. El procedimiento más utilizado fueron los colgajos pediculados (50\%), seguido por los colgajos locales $(41,2 \%)$ y, por último, se emplearon injertos de piel $(8,8 \%)$. Dentro de los colgajos pediculados, el más utilizado fue el colgajo antecubital (12 casos) con un $70,6 \%$, seguido por el colgajo braquial lateral con un $23,5 \%$ y, por último, con un solo caso, un colgajo propeller, que representó el 5,9\%. Dentro de las plásticas locales, los colgajos de avance representaron el $42,8 \%$, seguidos por las zetaplastias en un $35,7 \%$, los colgajos de transposición en un $14,3 \%$ y la w plastia en un $7,14 \%$. Todos los injertos de piel realizados fueron de espesor total. Las complicaciones fueron dehiscencia del borde del colgajo y epidermólisis. La ganancia objetiva de extensin en grados fue entre 5 y 80 grados.

Conclusiones. Podemos concluir que es posible la aplicación de nuestro algoritmo para el tratamiento en la secuela cicatrizar en codo. Siempre que sea posible, se recomienda utilizar un colgajo fasciocutáneo por su mejor cobertura, resistencia y poca morbilidad de obtención. Todo método quirúrgico debe acompañarse con medidas no quirúrgicas como la ferulización y la rehabilitación kinésica temprana para lograr un resultado sostenido en el tiempo.

Palabras claves: contractura posquemadura, codo, reconstrucción.

\begin{abstract}
Introduction. Burn injuries to the elbow can lead to significant functional impairment and major aesthetic problems. This represents a challenge for the plastic surgeon due to the variability in their complexity and presentation. The elbow can be constructed with a wide range of surgical technique such as skin grafts, local, regional or free flaps. However, contractures vary greatly, depending on the loss of function, depth and extension of tissue involvement, and duration of scarring and, therefore, surgical intervention should be tailored for each patient.

Objective. To present our experience and systematic approach for releasing postburn elbow contractures.

Materials and methods. A retrospective review of the cases of post-burn elbow contractures treated in our center between August of 2008 and August of 2018 was conducted.

Results. Since 2008 we have treated 34 patients. They included 20 female patients and 14 male patients. The average age was 21,2 years (range $3-50$ years) at the time of the first corrective operation. Severity of elbow contracture was graded as mild in $41,17 \%$ of the cases, moderate in $47,05 \%$ and severe in $11,7 \%$. Contractures were treated with fascio cutaneous pedicled flaps in $50 \%$ of the cases, with local flaps in $41,2 \%$ of the cases and with skin graft in $8,8 \%$.Of the fascio cutaneous flaps employed, 12 patients were treated with an antecubital flap, 4 with a lateral arm flap and 1 with a propeller flap. Among the local flaps, $z$ plasties and advancement flaps were the most commonly used. Elbow excursion improved in all cases between 5 and 80 degrees

Conclusions. Severe elbow contracture resulting from burn injury can be treated using a systematic approach according to the physical characteristics of the contracture. Although there are many techniques of soft-tissue coverage to treat elbow contractures, we advocate using well-vascularized fascio cutaneous flaps to provide coverage due to their resistance and low donor site morbidity.
\end{abstract}

Keywords: post-burn contractures, elbow, reconstruction.

REVISTA ARGENTINA DE CIRUGÍA PLÁSTICA 2020;26(2):68-75. HTTPS://DOI.ORG/10.32825/RACP/202002/0068-0075

\section{INTRODUCCIÓN}

Las retracciones cicatrizales secundarias a quemaduras representan un desafío para el cirujano plástico debido

1. Residente de Cirugía Plástica del Hospital de Quemados del Gobierno de la Ciudad de Buenos Aires.

2. Jefe de Unidad del Servicio de Cirugía Plástica del Hospital de Quemados del Gobierno de la Ciudad de Buenos Aires.

$\square$ Correspondencia: revista@sacper.org.ar

Los autores no declaran conflictos de intereses

Recibido: 10/02/2020 / Aceptado: 20/02/2020 a la variabilidad en la complejidad y presentación de las mismas. En el miembro superior, el codo es una de las articulaciones más afectadas, con una incidencia que oscila entre el 21 y el $42 \%{ }^{1}$.

Estudios biodinámicos muestran que el rango normal de flexoextensión del codo abarca de $0^{\circ}$ a $145^{\circ}$. A su vez, se acepta que el arco de movimiento mínimo para poder realizar actividades diarias es de $30^{\circ}$ a $130^{\circ}{ }^{2,3}$. La pérdida de movimiento del codo por debajo de estos rangos conllevan una limitación funcional y un impacto directo en la calidad de vida del paciente, impidiéndole realizar actividades básicas como comer, bañarse o asearse ${ }^{4}$, por lo que el tratamiento quirúrgico es imperativo. 


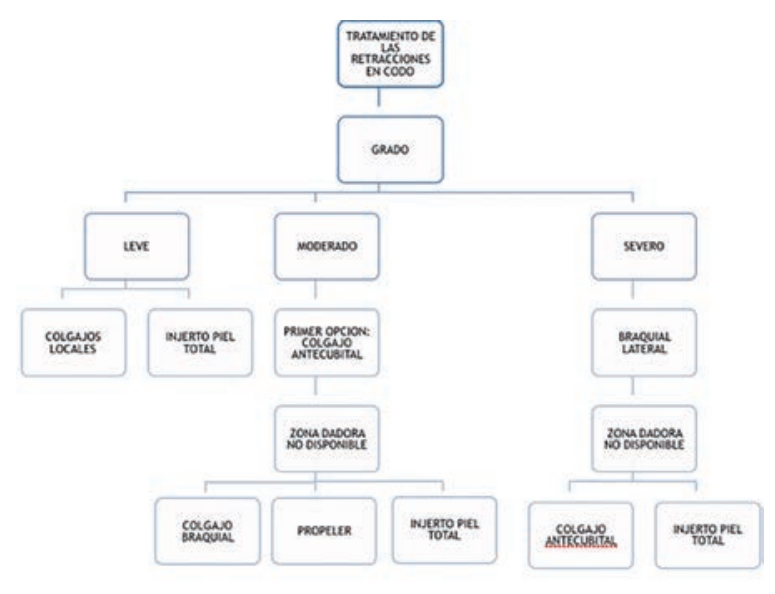

Figura 1. Algoritmo de tratamiento.

El objetivo principal de la reconstrucción consiste en restablecer la funcionalidad del codo, asegurando un amplio rango de movimientos. De ser posible, en un solo tiempo quirúrgico, con un tejido que posea características similares en cuando a textura, color y grosor, lo suficientemente fino y plegable para adaptarse al contorno de la fosa antecubital y con la menor morbilidad posible del sitio dador 5 .

Existen diferentes opciones descriptas en la literatura para el tratamiento de dichas secuelas, que incluyen desde injertos de piel total ${ }^{6,7}$, colgajos locales ${ }^{8-14}$, colgajos fascio-cutáneos ${ }^{15-18}$, colgajos musculares ${ }^{19-23}$, colgajos microquirúrgicos ${ }^{24-26} \mathrm{o}$ una combinación de ellos. Cada uno de estos presenta ventajas y desventajas. Sin embargo, la elección de la cobertura a utilizar dependerá de diferentes variables, tales como: el tamaño de la lesión, la exposición y compromiso de estructuras vitales, las comorbilidades del paciente, las zonas dadoras potenciales y la experiencia del equipo tratante, entre otras 5 .

La gran variabilidad de opciones quirúrgicas plantea la necesidad de sistematizar el tratamiento de las retracciones cicatrizales para facilitar la decisión terapéutica más adecuada para cada paciente. Por lo tanto, el objetivo de este estudio es la presentación de un algoritmo de tratamiento de la secuela posquemadura de codo basado en nuestra experiencia en el Servicio de Cirugía Plástica del Hospital de Quemados del Gobierno de la Ciudad de Buenos Aires.

\section{MATERIALES Y MÉTODOS}

Mediante un diseño observacional, descriptivo y retrospectivo, se realizó el presente trabajo sobre pacientes con diagnostico de retracción cicatrizal posquemadura en codo tratados en el Hospital de Quemados del Gobierno de la Ciudad de Buenos Aires, en el período comprendido entre agosto de 2008 y agosto de 2018. Se incluyeron en este estudio todos los pacientes entre 3 y 65 años que fueron sometidos a cirugías reconstruc- tivas para la liberación de contracturas del codo posteriores a una quemadura durante el período mencionado, con consentimiento informado. Se excluyeron del estudio aquellos pacientes que presentaron secuelas cicatrizales secundarias a trauma o tumores, pacientes con anquilosis o enfermedades degenerativas de las articulaciones y aquellos que no cumplieron con la rehabilitación kinésica correspondiente posoperatoria.

De los pacientes seleccionados, se realizó una revisión de las historias clínicas y se evaluaron los casos a través del examen físico y de la documentación fotográfica de los pacientes obtenida en los períodos pre- y posoperatorios, y se analizaron las diferentes variables: sexo, edad, comorbilidades, clasificación de gravedad, grado de retracción prey posoperatoria, tipo de reconstrucción empleada y complicaciones. Las variables mencionadas se reunieron en la Tabla 1, presentada en el apartado resultados.

Con el fin de estratificar las secuelas de quemaduras, se diseñó una clasificación basada en la disminución del arco de movimiento y en el porcentaje de piel afectada, que se expone en la Tabla 2.

El grado de retracción del codo se evaluó midiendo la pérdida de la extensión en grados a partir del rango fisiológico. Las complicaciones se dividieron en mayores y menores. Las primeras fueron aquellas que requirieron una nueva intervención quirúrgica, por ejemplo, una necrosis total del colgajo o un hematoma; las complicaciones menores fueron aquellas que no comprometieron la vitalidad del colgajo o del paciente y que pudieron resolverse mediante métodos conservadores, por ejemplo: seroma, dehiscencia, pérdida parcial de injertos o sufrimientos distales de colgajos.

\section{RESULTADOS}

Durante el período comprendido entre agosto de 2008 y agosto de 2018 se realizaron 34 procedimientos quirúrgicos para la reconstrucción de las secuelas cicatrizales en codo, todos de forma unilateral.

De los 34 pacientes operados, 20 eran del género femenino y 14 del género masculino, con edades que variaron desde los 3 hasta los 50 años, con una media de 21,2 años. De todos los pacientes, solo dos $(5,88 \%)$ presentaban antecedentes médicos de relevancia: tabaquismo y alcoholismo.

El 41,17\% (14 casos) presentaron una clasificación leve, el $47,05 \%$ (16 casos) una moderada y el 11,7\% (4 casos) una severa.

De todos los procedimientos quirúrgicos realizados, los colgajos pediculados fueron los más utilizados ( $50 \%$ de los casos), seguidos por los colgajos locales $(41,2 \%)$ y por último, se emplearon injertos de piel en un $8,8 \%$ de los casos.

Dentro de los colgajos pediculados, el más utilizado fue el colgajo antecubital (12 casos) con un $70,6 \%$, en segundo lugar el colgajo braquial lateral con un $23,5 \%$ y por último, con un solo caso, un colgajo propeller, que 


\begin{tabular}{|c|c|c|c|c|c|c|c|c|c|c|}
\hline Caso & Sexo & Edad & $\begin{array}{l}\text { Com or - } \\
\text { bi-lidades }\end{array}$ & Tipo & $\begin{array}{l}\text { Pérdida de la } \\
\text { ext. en grados } \\
\text { pre-Qx }\end{array}$ & Cirugía realizada & $\begin{array}{l}\text { Cierre de } \\
\text { zona dadora }\end{array}$ & $\begin{array}{l}\text { Pérdida de la } \\
\text { ext. en grados } \\
\text { post-Qx }\end{array}$ & $\begin{array}{l}\text { Mejoría } \\
\text { en grados }\end{array}$ & Complicaciones \\
\hline 1 & $\mathrm{~F}$ & 38 & & 3 & 69 & Braquial lateral & Injerto & 20 & 49 & \\
\hline 2 & $\mathrm{~F}$ & 37 & & 3 & 80 & Braquial lateral & Injerto & 15 & 65 & Necrosis \\
\hline 3 & $\mathrm{~F}$ & 45 & & 3 & 90 & Braquial lateral & Injerto & 10 & 80 & \\
\hline 4 & M & 7 & & 3 & 86 & Colgajo antecubital & Cierre & 15 & 71 & Dehiscencia \\
\hline 5 & M & 50 & & 2 & 40 & Braquial lateral & Injerto & 15 & 35 & Dehiscencia \\
\hline 6 & $M$ & 10 & & 2 & 40 & Colgajo antecubital & Cierre & 5 & 35 & \\
\hline 7 & $\mathrm{~F}$ & 29 & & 2 & 40 & Colgajo antecubital & Injerto & 5 & 35 & \\
\hline 8 & $\mathrm{~F}$ & 21 & & 2 & 45 & Colgajo antecubital & Cierre & 6 & 39 & \\
\hline 9 & $\mathrm{~F}$ & 16 & & 2 & 39 & Colgajo antecubital & Cierre & 8 & 31 & \\
\hline 10 & $\mathrm{~F}$ & 18 & & 2 & 40 & Colgajo antecubital & Injerto & 7 & 33 & \\
\hline 11 & $\mathrm{~F}$ & 3 & & 2 & 30 & Colgajo antecubital & Cierre & 4 & 26 & \\
\hline 12 & $M$ & 14 & & 2 & 40 & Colgajo antecubital & Cierre & 4 & 36 & \\
\hline 13 & $M$ & 49 & & 2 & 40 & Colgajo antecubital & Injerto & 5 & 35 & Dehiscencia \\
\hline 14 & M & 30 & & 2 & 30 & Colgajo antecubital & Injerto & 5 & 25 & \\
\hline 15 & M & 11 & & 2 & 45 & Colgajo antecubital & Injerto & 5 & 40 & \\
\hline 16 & $M$ & 9 & & 2 & 39 & Colgajo antecubital & Injerto & 5 & 34 & \\
\hline 17 & $\mathrm{~F}$ & 21 & & 2 & 40 & Colgajo propeller & Cierre & 5 & 35 & Epidermólisi \\
\hline 18 & $\mathrm{~F}$ & 20 & & 2 & 40 & Injerto & & 5 & 35 & \\
\hline 19 & M & 30 & Tabaq. & 2 & 40 & Injerto & & 5 & 35 & \\
\hline 20 & $M$ & 20 & & 2 & 45 & Injerto & & 5 & 40 & \\
\hline 21 & $\mathrm{~F}$ & 13 & & 1 & 10 & Colgajo de avance & & 5 & 5 & \\
\hline 22 & $\mathrm{~F}$ & 40 & & 1 & 5 & Colgajo de avance & & 0 & 5 & \\
\hline 23 & $\mathrm{~F}$ & 15 & & 1 & 5 & Colgajo de avance & & 0 & 5 & \\
\hline 24 & M & 18 & & 1 & 10 & Colgajo de avance & & 0 & 10 & \\
\hline 25 & $M$ & 14 & & 1 & 5 & Colgajo de avance & & 0 & 5 & \\
\hline 26 & $M$ & 15 & & 1 & 5 & Colgajo de avance & & 0 & 5 & \\
\hline 27 & $\mathrm{~F}$ & 31 & & 1 & 5 & Transposición & Cierre & 0 & & \\
\hline 28 & $\mathrm{~F}$ & 18 & & 1 & 15 & Transposición & Cierre & 0 & 15 & \\
\hline 29 & $\mathrm{~F}$ & 29 & & 1 & 10 & W-plastia & & 0 & 10 & Cicatriz retráctil \\
\hline 30 & $\mathrm{~F}$ & 18 & & 1 & 10 & Zetaplastia & & 0 & 10 & \\
\hline 31 & $M$ & 43 & Alcohol. & 1 & 10 & Zetaplastia & & 0 & 10 & \\
\hline 32 & $\mathrm{~F}$ & 17 & & 1 & 10 & Zetaplastia & & 0 & 10 & \\
\hline 33 & $\mathrm{~F}$ & 21 & & 1 & 5 & Zetaplastia & & 0 & 5 & \\
\hline 34 & $\mathrm{~F}$ & 22 & & 1 & 5 & Zetaplastia & & 0 & 5 & \\
\hline
\end{tabular}

representó el 5,9\%. Dentro de las plásticas locales, los colgajos de avance representaron el 42,8\%, seguidos por las zetaplastias en un $35,7 \%$, los colgajos de transposición en un $14,3 \%$ y la w plastia en un 7,14\%.

Todos los injertos de piel realizados fueron de espesor total. Se observaron complicaciones en un $14,7 \%$ de los casos. En tres pacientes se evidenció una dehiscencia del colgajo y en uno una epidermólisis parcial del colgajo, cuyo tratamiento fue conservador. En uno de los pacientes, se observó la pérdida total del colgajo que se trató con toilettes quirúrgicas y autoinjerto.

La ganancia objetiva de extensión en grados fue entre $5^{\circ}$ y $80^{\circ}$ siendo mayor cuando más severa fuese la clasificación.

En la Tabla 1 se muestran todos los datos expuestos en el apartado anterior y en la Figura 1 se puede observar el algoritmo de tratamiento basado en nuestra experiencia. A continuación se presenta una serie de casos representativos de la muestra.

\section{Caso 1}

Paciente de 43 años, con retracción cicatrizal posquemadura grado I de larga data en donde se observa brida
TABLA 2. Clasificación según disminución del movimiento y porcentaje de piel afectada.

\begin{tabular}{|c|c|c|}
\hline Retracción & $\begin{array}{c}\text { Grados de pérdida } \\
\text { de la extensión }\end{array}$ & $\begin{array}{c}\text { Compromiso de la superfice } \\
\text { de la fosa antecubital }\end{array}$ \\
\hline Leve & $<20^{\circ}$ & Brida cicatrizal \\
\hline Moderada & $20^{\circ}$ a $50^{\circ}$ & Menos del $50 \%$ \\
\hline Severa & $>50$ & Más del $50 \%$ \\
\hline
\end{tabular}

en cara interna de codo. Se realizó una cadena de zetaplastias (Figuras 2 y 3 ).

Caso 2

Paciente de 49 años, con una clasificación moderada de contractura cicatrizal de larga data en donde se realizó un colgajo antecubital en isla y cierre de la zona dadora con injerto de piel total (Figura 4 a 7).

\section{Caso 3}

Paciente de 7 años con retracción cicatrizar posquemadura grave. Se realizó un colgajo antecubital al no contar con zona dadora disponible para colgajo braquial lateral (Figuras 8 a,1 1). 


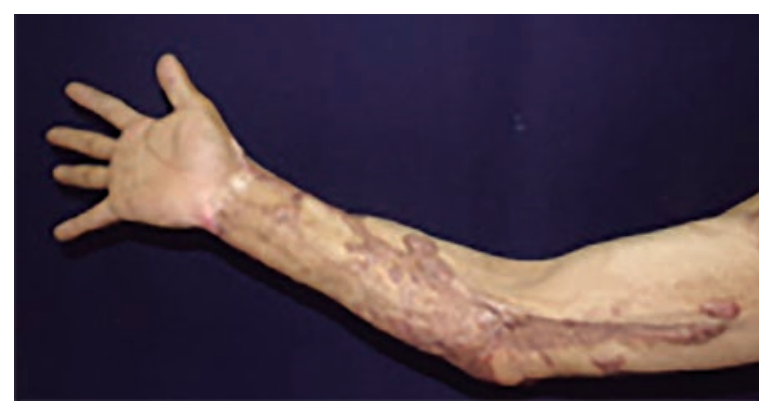

Figura 2. Vista preoperatoria.

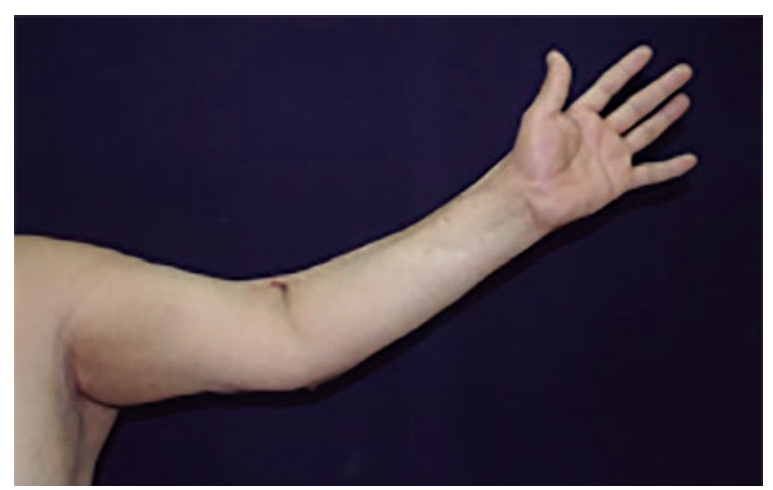

Figura 4. Vista frontal preoperatoria.

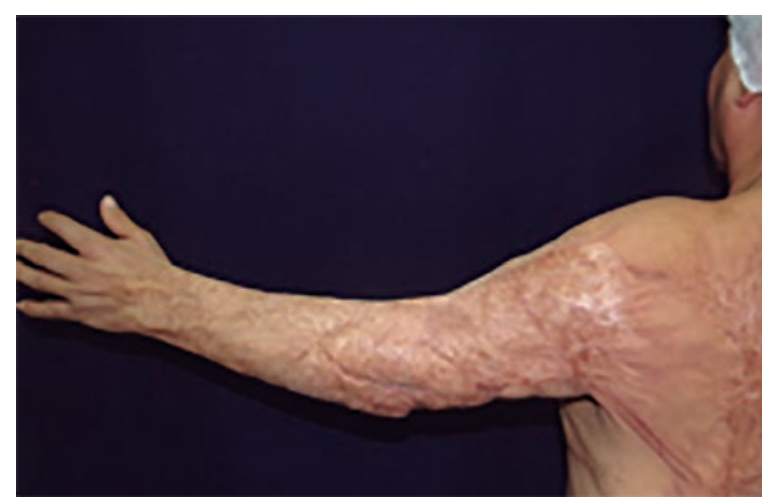

Figura 6. Vista posterior posoperatoria.

\section{Caso 4}

Paciente de 50 años con cicatriz retráctil severa. Se le realizó un colgajo braquial lateral a flujo reverso en isla con cierre de la zona dadora con injerto de piel (Figuras 12 a 16).

\section{DISCUSIÓN}

Las retracciones cicatrizales representan una causa de morbilidad en los pacientes que sufren quemaduras y tienen un impacto negativo en la calidad de vida y en la habilidad para realizar actividades cotidianas como comer o higienizarse, representando un motivo de consulta frecuente para el cirujano plástico.

El objetivo principal de la reconstrucción consiste en restablecer la funcionalidad del codo, asegurando un amplio rango de movimientos con un tejido de similares características y con mínima morbilidad de la zona dadora.

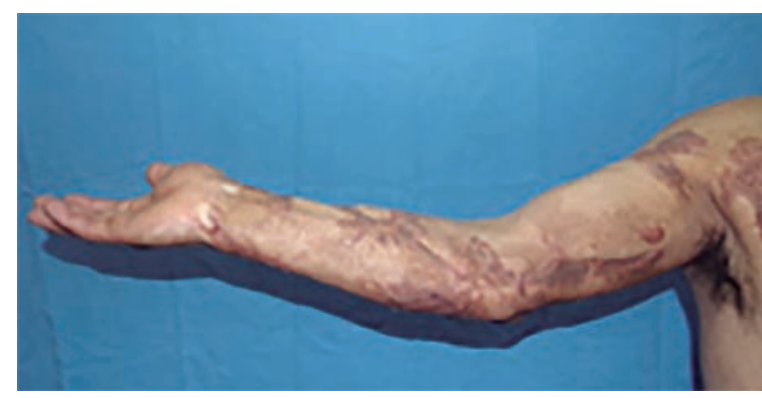

Figura 3. Vista a los 3 meses postoperatoria.

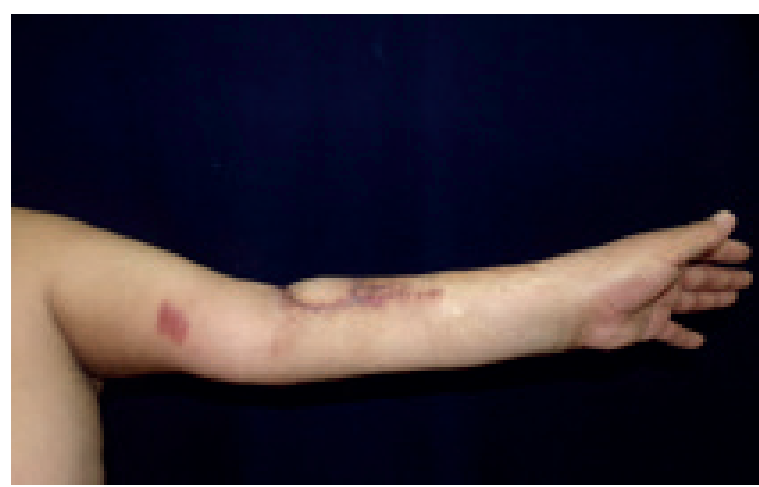

Figura 5. Vista frontal al mes posoperatorio.

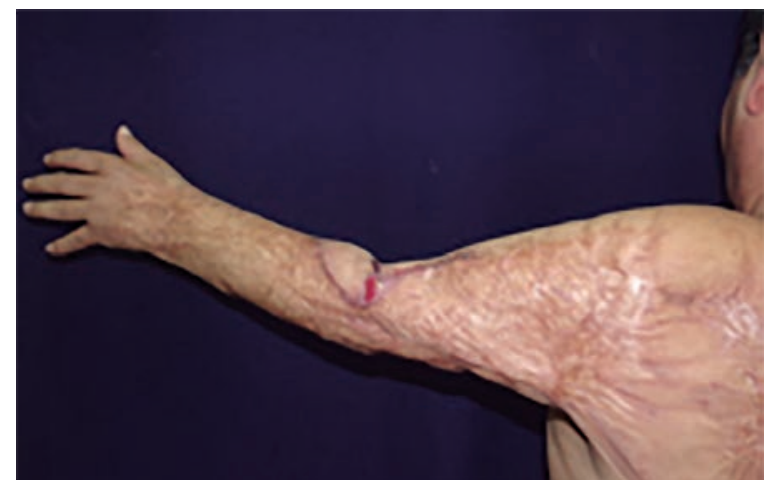

Figura 7. Vista posterior posoperatoria.

Existen diferentes tratamientos descriptos en la literatura para la reconstrucción de secuelas cicatrizales en codo, cada una con ventajas y desventajas.

Los injertos de piel total pueden ser utilizados para defectos de cualquier tamaño que posean un lecho receptor apto y son una opción razonable en pacientes con muchas comorbilidades en quienes un colgajo no es una opción viable. Sin embargo, requieren un lecho receptor bien vascularizado y ferulizaciones prolongadas para evitar la contractura secundaria ${ }^{27}$. Además, el injerto no provee una cobertura duradera apropiada que resista los movimientos de flexión y extensión repetidos, lo que puede llevar a ulceraciones o erosiones frecuentes $^{28}$.

Los colgajos locales son útiles para el tratamiento de secuelas cicatrizales leves o bridas con tejido adyacente sano disponible. Son colgajos random que se nutren a través de los plexos subdérmicos y dérmicos. Tienen 


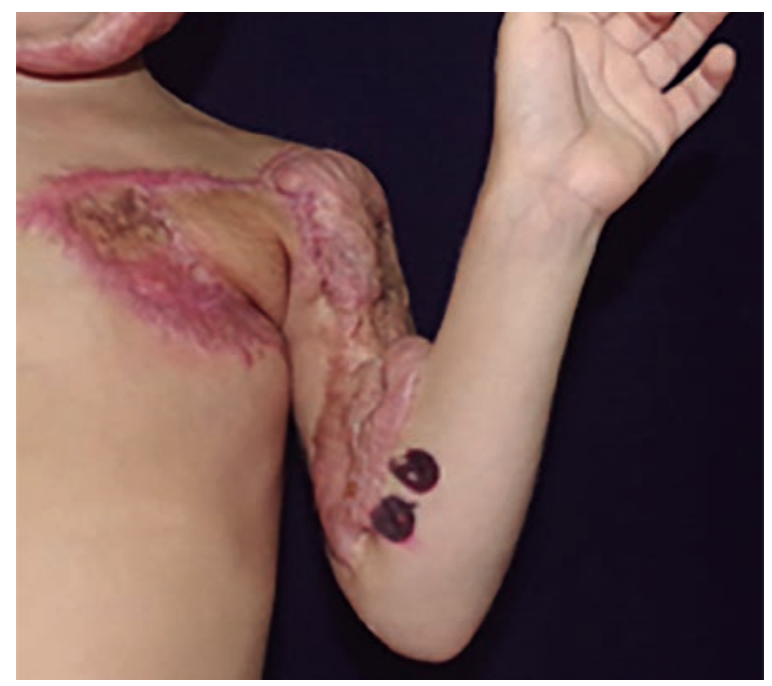

Figura 8. Vista anterior preoperatoria.

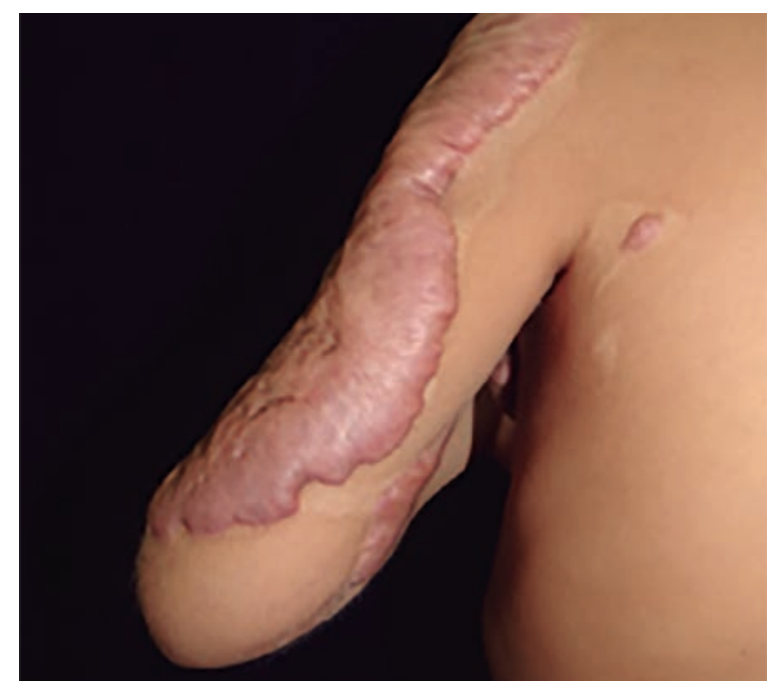

Figura 9. Vista posterior preoperatoria.

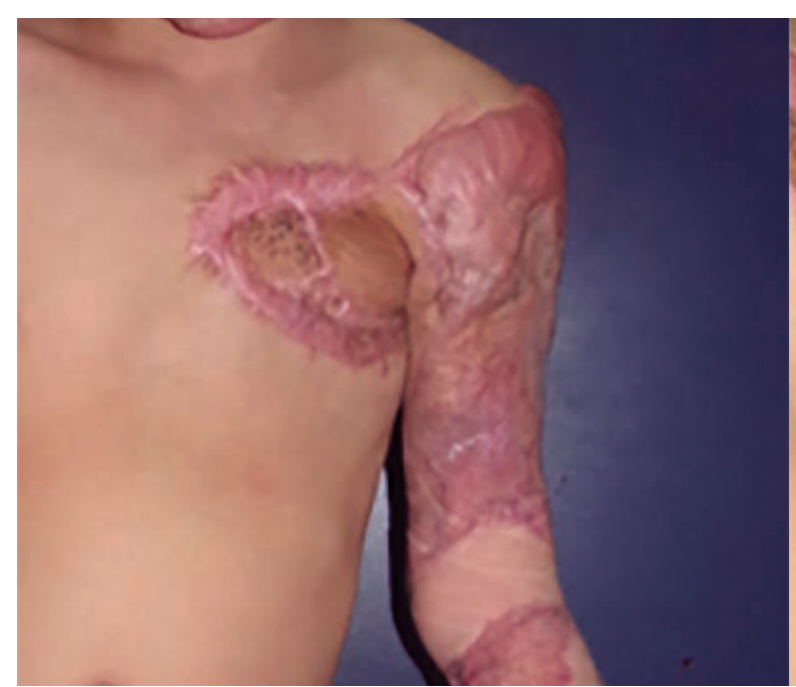

Figura 10 y 11. Vista frontal a los 3 meses posoperatorio.

la ventaja de aportar tejido con similares características en cuanto a textura y coloración, de generar baja morbilidad y de la facilidad en la obtención. Sin embargo, aportan poco tejido y son limitados. Dentro de las diferentes opciones, las zetaplastias son utilizados para la liberación de cicatrices lineales con tejido sano adyacente, generalmente con ángulos de alrededor de 60 grados. En nuestro estudio, se utilizaron zetaplastias incluso en pacientes con tejido cicatrizal adyacente de buena textura y elasticidad, logrando liberar la brida retráctil y obteniendo buen resultado funcional de todas formas.

Los colgajos musculares brindan buena cobertura vascular que resiste a contracturas secundarias, pero tienden a dar un aspecto abultado al área antecubital ${ }^{29}$. A su vez, los colgajos musculares regionales, como el del ancóneo, pueden estar comprometidos por la quemadura al estar adyacentes a la lesión.

Los colgajos microquirúrgicos son una excelente opción para el tratamiento de las secuelas en codo y hoy en día se los considera como una de las primeras líneas de tratamiento. Sin embargo, requieren experiencia, recursos y mayor tiempo operatorio.

Los colgajos que se prefieren en nuestro servicio son los colgajos pediculados fasciocutáneos. Uno de los colgajos más utilizados en nuestro Hospital para la resolución de las secuelas cicatrizales fue el colgajo antecubital, descripto por Cormack y Lamberty en $1983^{30}$. Se trata de un colgajo fasciocutáneo axial basado en la arteria cubital inferior, rama de la arteria radial en la mayoría de los $\operatorname{casos}^{31}$, que emerge a $4 \mathrm{~cm}$ del pliegue antecubital y que se puede utilizar para cubrir defectos menores y moderados del tercio proximal del antebrazo incluyendo la cara anterior, medial y lateral del codo, pudiendo llegar incluso a cubrir defectos periolecranianos ${ }^{32}$.

Se puede realizar a pedículo cutáneo o en isla para evitar la "oreja de perro". Lamberty y Cormack describieron también la posibilidad de utilizarlo como un colgajo libre. Sin embargo, la necesidad de incluir parte de 


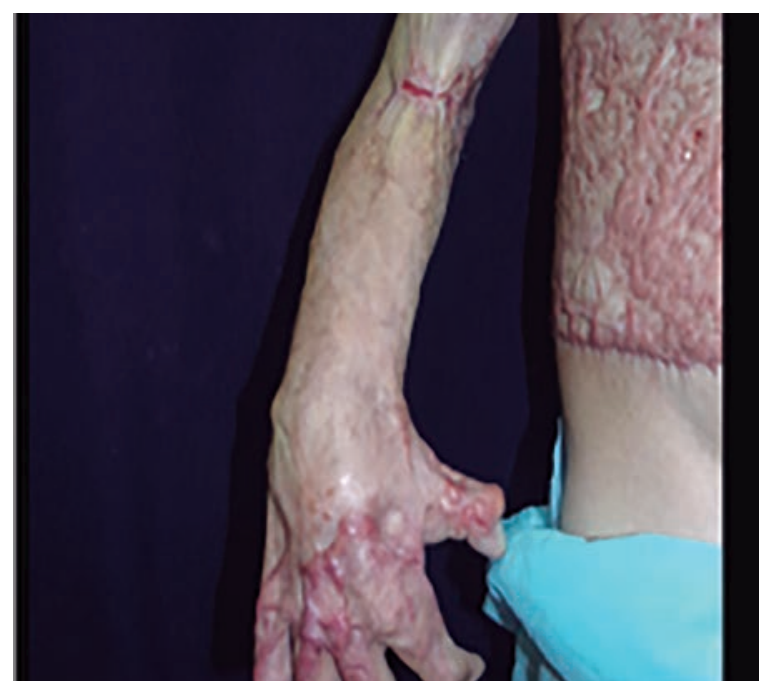

Figura 12. Vista anterior preoperatoria.

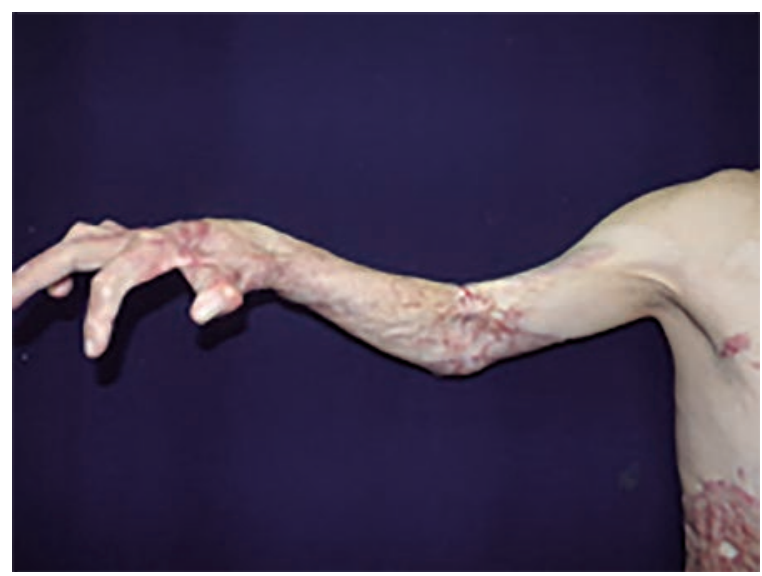

Figura 14. Vista máxima extensión de codo

la arteria radial para obtener un vaso adecuado para la anastomosis, la variabilidad de su origen y los nuevos colgajos libres que surgieron hacen que no sea una opción fiable ${ }^{33}$.

Si el ancho del colgajo no supera los $4 \mathrm{~cm}$, se puede realizar un cierre primario. De lo contrario, se realizará el cierre de la zona dadora con un injerto, de ser posible, con piel total. Este colgajo presenta varias ventajas, a saber: no utiliza un vaso mayor del antebrazo conservando así los dos pedículos principales de la mano, tiene poca morbilidad de la zona dadora si el cierre se realiza de forma primaria, es de fácil disección con una baja curva de aprendizaje. Una de las limitantes del colgajo es la dependencia de la zona dadora. En nuestro estudio, el colgajo antecubital resultó ser un colgajo seguro con un bajo índice de complicaciones, las cuales fueron todas menores (dehiscencia), con un resultado favorable a largo plazo y baja morbimortalidad.

En los casos de retracciones más severas o en aquellas retracciones moderadas que no tuvieran disponibilidad de zona dadora en antebrazo, la opción más utilizada fue el colgajo braquial lateral a pedículo reverso. Este colgajo fue descripto por Song et al. en 1982 como un colgajo libre ${ }^{26}$. En 1984, Katsaros et al., en un in-

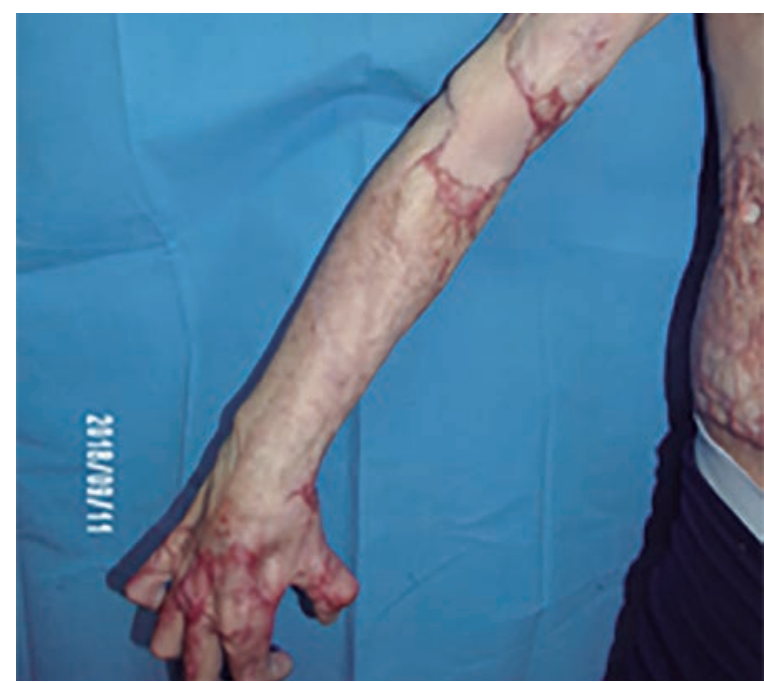

Figura 13. Vista anterior a los 6 meses posoperatorio.

tento por encontrar un colgajo con pedículo constante en el brazo en contrapartida del braquial interno, realizaron estudios y describieron detalladamente la anatomía del colgajo braquial lateral y sus diversas aplicaciones clínicas ${ }^{34}$. En 1987, Culbertson et al, publicaron el uso del colgajo braquial lateral en forma reversa para la reparación de defectos en el codo, aprovechando la anastomosis que hay entre la arteria colateral radial posterior y la recurrente interósea posterior ${ }^{27}$.

La anatomía de este colgajo esta bien descripta en la literatura $^{35-37}$. Se trata de un colgajo fasciocutáneo basado en ramas septocutáneas de la arteria colateral radial posterior (PRCA). La arteria humeral profunda discurre por el canal radial en la cara posterior del húmero para ingresar en el septum intermuscular lateral. Aquí se divide en dos ramas terminales. El ramo anterior es la arteria radial colateral anterior, ramo menor e inconstante, que acompaña el recorrido del nervio radial entre el braquiorradial y el músculo braquial anterior para terminar anastomosándose con la arteria recurrente radial, rama de la arteria radial. La arteria radial colateral posterior es la rama más importante y que parece continuar el trayecto de la arteria humeral profun$\mathrm{da}$ en el septum intermuscular entre el tríceps y el braquiorradial. Este vaso envía diferentes perforantes septocutáneas que irrigan la piel de la cara lateral del brazo. La PRCA continúa por detrás del epicóndilo y se anastomosa con la arteria recurrente interósea o recurrente radial posterior.

Por lo tanto, el colgajo braquial lateral reverso puede basarse en la anastomosis de la colateral radial anterior y la radial recurrente como fue reportado por Maruyama y Takeuchi ${ }^{38}$, o puede basarse en la anastomosis de la PRCA con la interósea recurrente como lo describió Culberston.

Este colgajo puede cubrir defectos de hasta $15 \mathrm{~cm}$ y si se realiza con un ancho de hasta $6 \mathrm{~cm}$, puede cerrarse de forma primaria. De lo contrario será necesario un injerto de piel o expandirlo previamente. Las ventajas 


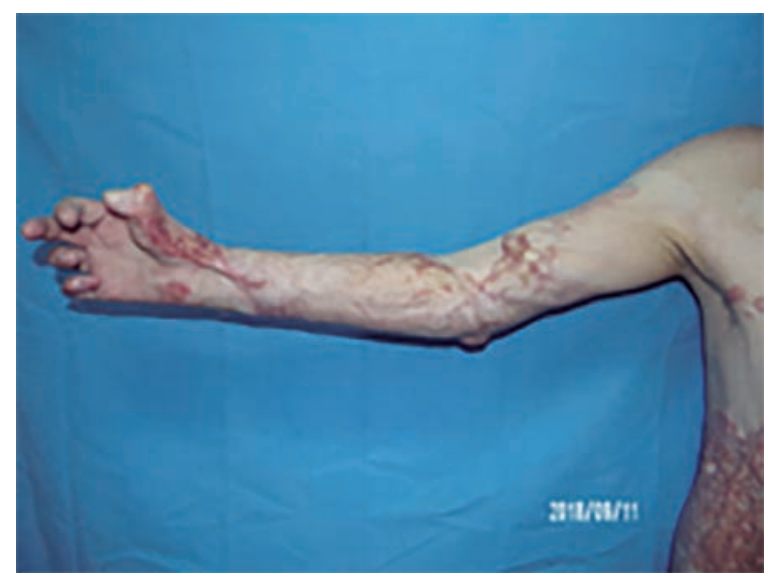

Figura 15. Máxima extensión de codo a los 6 meses posoperatorio.

de este colgajo incluyen una anatomía constante, la no necesidad de sacrificar una arteria mayor, un cierre directo de la zona dadora con baja morbilidad en el caso que se realizara de $6 \mathrm{~cm}$ o menos y que puede obtenerse de forma compuesta si se necesita. Por otro lado, el colgajo deja una importante cicatriz visible en el brazo y, si se realiza el cierre con injerto, un defecto inestético, tiende a ser un colgajo abultado, es más difícil su disección por el riesgo de lesionar el nervio radial, transporta pelo a una zona por lo general lampiña y suele afectar la sensibilidad del antebrazo.

Sin embargo, es un colgajo fiable que puede resolver secuelas graves en casos en donde no se cuenta con la posibilidad de realizar un colgajo microquirúrgico. A su vez, algunos riesgos como el de la lesión del nervio radial pueden disminuirse si se diseca en un plano suprafascial basándose en una arteria perforante previamente identificada por Doppler.

En nuestra experiencia, este colgajo nos permitió cubrir defectos mayores del codo sin necesidad de recurrir a un colgajo microquirúrgico. Es un colgajo con mayor número de complicaciones y con un resultado

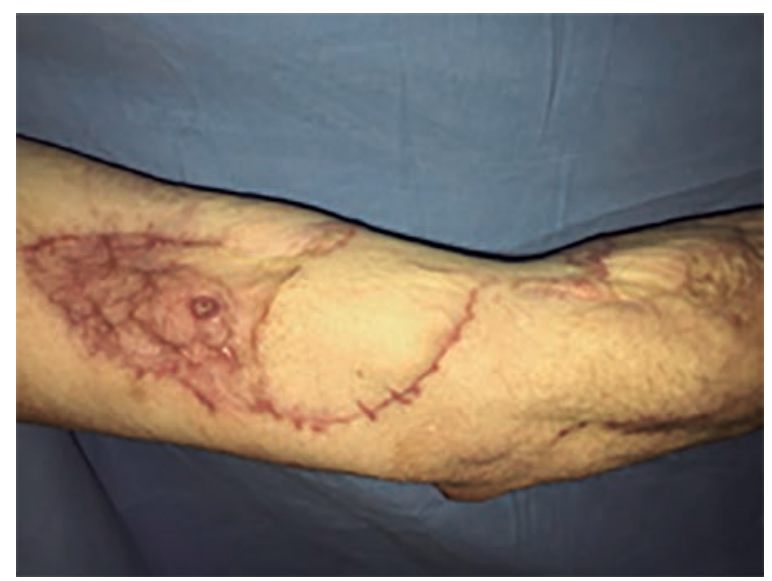

Figura 16. Vista lateral mostrando el colgajo y la zona dadora.

estético más pobre pero, a su vez, muy útil para cuando no se dispone de tejido sano en el antebrazo para realizar plásticas locales o un colgajo antecubital.

Independientemente del colgajo utilizado, es importante combinar las técnicas quirúrgicas con los métodos no quirúrgicos como son la inmovilización en hiperextensión con férula del miembro superior durante la primera semana y luego la elastocompresión y la rehabilitación kinésica temprana para lograr el mejor resultado a largo plazo.

\section{CONCLUSIÓN}

Podemos concluir que es posible la sistematización de nuestro algoritmo para el tratamiento en la secuela cicatrizar en codo.

Siempre que sea posible, se recomienda utilizar un colgajo fasciocutáneo por su mejor cobertura, resistencia y facilidad de obtención.

Todo método quirúrgico debe acompañarse con medidas no quirúrgicas como la ferulización y la rehabilitación kinésica temprana para lograr un resultado sostenido en el tiempo.

\section{BIBLIOGRAFÍA}

1. Schneider JC, Holavanahalli R, Helm P, Goldstein R, Kowalske K. Contractures in burn injury: Defining the problem. J Burn Care Res 2006:27:508-14.

2. Morrey BF, Askew LJ, Chao EY. A biomechanical study of normal functional elbow motion. J Bone Joint Surg Am 1981;63:872-7.

3. SchrumpFMA, Lyman S. Incidence of postoperative elbow contracture release in New York State. J Hand Surg Am 2013 Sep;38(9):174652.

4. Leblebici B, Adam M, BağişS, et al. Quality of life after burn injury: the impact of joint contracture. J Burn Care Res 2006;27:864-8.

5. Choudry UH, Moran SL, Li S, Khan S. Soft-Tissue Coverage of the Elbow: An Outcome Analysis and Reconstructive Algorithm. Plast Reconstr Surg 2007 May; 119(6):1852-7.

6. Iwuagwu FC, Wilson D, Bailie F. The use of skin grafts in postburn contracture release: a 10-year review. Plast Reconstr Surg 1999:103:1198-204.
7. Stern PJ, Law EJ, Benedict FK, et al. Surgical treatment of elbow contractures in postburn children. Plast Reconstr Surg 1985; 76:441-6.

8. Suzuki S, Um SC, Kim BM, Shin-ya K, Kawai K, Nishimura Y. Versatility of modified planimetric Z-plasties in the treatment of scar with contracture. Br J Plast Surg 1996;51:363-9.

9. CooperMA. Multiple Y-V-plasty in linear burn scar contracture release. Br J Plast Surg 1990;43:145-9.

10. Vartak A, Keswani MN. X-plasty for repair burns contractures. Burns 1992;18:326-8.

11. Suliman MT. Experience with seven flap-plasty for the release of burn contractures. Burns 2004;30:374-9.

12. Hyakusoku H, Fumiiri M. The squere flap method. Br J Plast Surg 1987:40:40-6.

13. Grishkevich VM. The post-burn elbow medial flexion scar contracture treatment with trapeze-flap plasty. Burns 2009;35:280-7. 
14. Aslan G, Tuncali D, Cigsar B, Barutcu AY, Terzioglu A. The propeller flap for postburn elbow contractures. Burns 2006;32:112-5.

15. El-Khatib HA, Mahboub TA, Ali TA. Use of an adipofascial flap based on the proximal perforators of the ulnar artery to correct contracture of elbow burn scars: An anatomic and clinical approach. Plast Reconstr Surg 2002;109:130-6.

16. Lai CS, Tsai CC, Liao KB, Lin SD. The reverse lateral arm adipofascial flap for elbow coverage. Ann Plast Surg 1997;39:196-200.

17. Lamberty BG, Cormack GC. The antecubital fasciocutaneous flap. Br J Plast Surg 1983;36:428-33.

18. Zancolli EA, Angrigiani C. Posterior interosseous island forearm flap. $J$ Hand Surg (Br.) 1988;13:130.

19. Rohrich RJ, Ingram AE. Brachioradialis muscle flap: Clinical anatomy and use in soft-tissue reconstruction of the elbow. Ann Plast Surg 1995;35:70-6.

20. Wysocki RW, Gray RL, Fernandez JJ, Cohen MS. Posterior elbow coverage using whole and split flexor carpi ulnaris flaps: A cadaveric study. J Hand Surg Am 2008;33:1807-12.

21. Chang $L D$, Goldberg NH, Chang B, Spence R. Elbow defect coverage with a one-staged, tunneled latissimus dorsi transposition flap. Ann Plast Surg 1994;32:496-502.

22. Uygur F, Sever C, Tuncer S, Alagoz S. Reconstruction of postburn antebrachial contractures using pedicled thoracodorsal artery perforator flaps. Plast Reconstr Surg 2009;123:1544-52.

23. Schmidt CC, Kohut GN, Greenberg JA, Kann SE, Idler RS, Kiefhaber $T R$. The anconeus muscle ap: Its anatomy and clinical application. $J$ Hand Surg Am 1999;24:359-69.

24. Derderian CA, Olivier WA, Baux G, Levine J, Gurtner GC. Microvascular free-tissue transfer for traumatic defects of the upper extremity: A 25-year experience. J. Reconstr. Microsurg 2003;19:455.

25. Jones NF, Jarrahy R, Kaufman MR. Pedicled and free radial forearm aps for reconstruction of the elbow, wrist, and hand. Plast Reconstr Surg 2008;121:887-98.
26. Song $R$, Song $Y, Y u Y$. The upper arm free ap. Clin Plast Surg 1982;9:27-35.

27. Culbertson J, Mutimer $K$. The reverse lateral arm flap for elbow coverage. Ann Plast Surg 1987;18:1.

28. Stevanovic M, Sharpe F. Soft-Tissue Coverage of the Elbow. Plast Reconstr Surg 2013;132(3).

29. Kung T, Jebson P. An individualized approach to severe elbow burn contractors. Plast Reconst Surg 2012;129(4).

30. Lamberty BG, Cormack GC. The antecubital fasciocutaneous flap. Br J Plast Surg 1983;361414:428-33.

31. Cormack and Lamberty. The arterial anatomy of skin flaps. 2nd ed. Edinburgh: Churchill Livingston, 1994.

32. Hierner R, Putz AT, Bishop Z, Shen K. Wilhel27m. Flaps in Hand and Upper Limb Reconstruction. 1st edition. Elsevier $\mathrm{GmbH}$, Munich, Germany, 2014.

33. van Landuyt K, De Cordier BC, Monstrey S, Blondeel PN, Tonnard $P$, Verpaele $A$, Matton $G$. The antecubital fasciocutaneous island flap for elbow coverage. Ann Plast Surg. 41 (1998) 252257.

34. Katsaros J, Schusterman M, Beppu M, Banis JC, Acland RD. The lateral upper arm flap: anatomy and clinical applications. Ann Plast Surg 1984:12:489-500.

35. Cormack GC, LambertyBG. Fasciocutaneous vessels in the upper arm: application to the design of new fasciocutaneous flaps. Plast Reconstr Surg 1984;74:244-9.

36. Rivet $D$, Buffet $M$, Martin $D$ et $n l$. The lateral arm flap: an anatomic study. J Reconstr Microsurg 1987;3:121-32.

37. Yousif NJ. Warren R. Matloub HS. Sanuer JR. The lateral arm fascia free flap: its'anatomy and use \& reconstruction. Plast Reconstr Surg 1990;86:1138-45.

38. Maruyama Y. Takeuchi S. The radial recurrent fasciocutaneous flap: reverse upper arm flap. Br J Plast Surg 1986;39:458861. 